\title{
Design and Implementation of Cluster Evolution based
}

\author{
Mobility Model
}

\author{
Dong Wang, Zhouli Wang, Xiaohong Li, Zhu xiao \\ College of Information Science and Engineering \\ Hunan University \\ Changsha, China \\ wangzhouli@hnu.edu.cn
}

\begin{abstract}
The movement of nodes has a great influence on the performance of protocols in mobile ad hoc networks. Mobility model is used to describe the movement of nodes, react the mobile characteristics really. In real circumstances, mobile devices are usually attached to humans, so the movement of such devices has the movement features of human movement. Through analyzing the cluster evolution features of real GPS trace, this paper proposed a cluster evolution based mobility model(CEMM), which reflects the human cluster dynamics features in real urban environment. We also compared the mobility features of CEMM with the classical human mobility features model SMOOTH, the results show CEMM works better in reflecting the cluster dynamics features.
\end{abstract}

Keywords: Mobile Ad Hoc network; Mobility model; Human Mobility; GPS data analysis

\section{INTRODUCTION}

Mobile ad-hoc networks (MANET) is a kind of multiple hops, self-organization wireless communication network. Due to it does not depend on any infrastructure network, MANET is widely used in military field, earthquake relief, and other areas of the emergency communications. In MANET networks, mobility as one of the important characteristics has an impact on change of network topology, network capacity [1],routing performance [2] and cluster maintain [3]. Therefore, the performance of MANET routing relies on the movement of wireless carriers. Most of the mobile device is carried by the people, so the understanding of human movement behavior is helpful to the research of MANET protocol performance [10].

Due to the cost of large-scale deployment of nodes in the real environment to construct MANET is too expensive, the current MANET protocol development and evaluation depend on simulation. As an important part of the simulation tool, mobility model used to describe the movement of network nodes. An ideal mobility model requires the following two characteristics: (1) reliability, the most important characteristics of mobility model is a real reliability, only a true description of the moving characteristics of nodes, the results of simulation can reflect the real performance of the protocol; (2) simple and easy to operate, there are few parameters and easy to use. As developers use tools, mobility model should not be too complicated.

The main contribution of this paper is proposed a kind of mobility model based on cluster evolution. In the mobility model which based on human movement characteristics, seldom consider cluster evolution regularity of human movement. Through the analysis of Shanghai city taxi data [4], we build a mobility model based on the set of cluster evolution., and compare it with human mobility model smooth. The simulation results shows that the proposed model based on cluster evolution mobile possess the above characteristics and smooth model does not fully comply with the above characteristics.

In this paper, the following content is as follows: the second section introduces related work; Section 3 introduces the establishment of the model based on cluster evolution; Section4 presents the experimental verification; Section 5 summarizes the full paper.

\section{RELATED WORK}

Mobility model can be divided into two categories as introduced above. The earliest and most widely used synthetic mobility model is random mobility model, which 
included Random Way Point mobility model, Random Direction mobility model, Random Walk mobility model, etc. But, random mobility model lacks the necessary truth because the random movement rule it used does not match the reality. To compensate for the unreal situation of node suddenly changes its speed, direction, it was proposed the time correlation mobility model, which node moves associated with the history of its mobile state. For example, the mobile node has gliding property in Gauss-Markov mobility model [6].Considering the surrounding nodes affected the mobile node, somebody proposed spatial correlation mobility model, such as Reference Point Group Mobility model, which describes the random movement of a set of nodes and the individual node in the group. The environment of the simulation scenario affected the mobile node in the location limited mobility model, such as setting obstacles, roads, etc in the simulation scenario. Manhattan Mobility Model and Obstacle Mobility Model are the representative models of location limited mobility model.

Trace based mobility model is widely researched, which is established by analyzing collected trajectory data in real environment and extracting the mobile features of nodes. Therefore, the trajectory analysis based mobility model is more reliable. In [5], current trace analysis based mobility model is summarized. As most of the communication equipment carried by a person, many research of mobility model is concentrated on the mobile features of human now. The study found the difference between characteristics of human movement extracted after processing real data sets and the traditional random mobility model, which confirms that human movement is not the real sense of random motion [11]. For example, Hotspot Gravitation Mobility Model [7] describes that humans tend to move around in the hot zone. SLAW [8] model shows communication interval and moving path have truncated power-law distribution. However, the model is too complex to be implemented. SMOOTH [9] simplified the implementation process of SLAW and the same effect is achieved.In [4], the cluster evolution of human movement is achieved by analyzing taxi trajectory data sets of Beijing city and Shanghai city. There is no mobility model does a study on the cluster evolution characteristic of human movement so far.

\section{Cluster Evolution BASEd Mobility MODEL}

The main work of this paper is to analyze the cluster evolution character result of the taxi's GPS trajectory data, to establish the cluster evolution mobility model, making its generation trajectory to have the same cluster evolution character, and compare it with the true GPS trajectory data of taxi and the generation trajectory data of smooth model to verify the authenticity of the mobility model proposed by this paper.

In [4] obtains the cluster evolution character through analyzing Shanghai GPS dataset: a. In each time point, the complementary cumulative distribution function of the cluster size submits to the power-law distribution; b. Cluster division inclines to small cluster, cluster combination inclines to big cluster swallowing small cluster; c. Node number of the cluster sustains rather stable, that is the node number of joining in the cluster and leaving the cluster is roughly equal; d. Complementary cumulative distribution of the cluster's survival time submits to the power-law distribution.

Smooth is a kind of rather classical mobility model used to study the human mobility discipline in nowadays. However, many aspects of smooth model don't accord with the cluster evolution discipline. For example, complementary type distribution of the cluster size doesn't submit to the power-law distribution, node number of the cluster is becoming less and et al. while this paper proposes a kind of mobility model based on the cluster evolution discipline, on the basis of meeting the above 4 items of cluster evolution discipline, try to make the realization process of the model easy and convenient to use.

This chapter mainly introduces the realization process of the mobility model based on the cluster evolution. The design of mobility model is usually divided into several steps as initial setup, node destination chosen, and node moving and et al. The realization of mobility model is presented as follows specifically.

\section{A. Initialize settings}

First of all, it is the initialization of the simulation area, which is a clear two-dimensional space adopted by this paper, with its length xRange, width yRange. in order to make the realization of mobility model easier, the scene of mobility model will be divided into the shape of grid in 
this paper. The grid is composed of two kinds: dissociative grid and cluster grid. When the number of grid reaches the minimum number of the cluster, then the grid is called cluster grid, otherwise, it will be called dissociative grid.

In the initialization process, we need to confirm the node number of the cluster at first. The proportion of cluster node to the total nodes number is called $\alpha$, by analyzing the dataset it is found that $\alpha$ usually locates between $20 \%$ to $30 \%$. We confirm the location of the initial cluster grids through random selection, and we will deploy the cluster nodes to the cluster grids by the following proportion:

(1) Small cluster: the node number is $5-10$, the proportion is $90 \%$.

(2) General cluster: the node number is 11-30, the proportion is $9.7 \%$.

(3) Big cluster: the node number is above 30 , the proportion is $0.3 \%$.

Every cluster has two properties: the Lifetime and the Attract. The lifetime is from the time of the cluster's generation to the present time, the attract is the attract degree of the cluster to certain node, which is related with the distance, lifetime, and the present node number of cluster. The formula of the attract will be specified in the back of this paper.

\section{B. The selection of the destination}

The selection of the destination is critical to de design of the mobility model, which describes the concrete moving process in the simulation. When selecting the destination of node, we need to confirm the type of the target grids, cluster grids or dissociative grids. For example, when the dissociative node selects the destination, we judge whether the target grids are the cluster girds by the probability $\beta$, otherwise the grids will be the dissociative grids. Similarly, we judge whether the cluster node has changed into dissociative node by the same probability, according to this, to maintain the dynamic equilibrium of the cluster node's proportion in the mobility model.

After confirming the type of the target grids, we need to confirm which grid is to be chosen to the target grid. First of all, introduce the selection method of dissociative grids. According to the [8] that the distance of straight line moving by human submits to the power-law distribution, so in this paper, the selection proportion of the dissociative grids is related to the distance between the node to the grid, and the nearer the distance is, the greater the selection proportion is.

The selection method of the cluster grid: when the node has confirmed the cluster grid as the target grid, first of all, all cluster grids' attract to the node needs to be calculated, and take the cluster grid which has the greatest attract as the target grid. According to the analysis result of the GPS dataset: 1. The node is inclined to join in the nearby cluster grid; 2 . The node is inclined to join the small cluster and the general cluster; 3 . The node is inclined to join in the newly-generated cluster. So the formula of the attract is as follows:

$$
\text { Attrack }=a * \frac{1}{\sqrt{\text { Dis } \tan c e}+u}+b * \frac{1}{\sqrt{\mid \text { Nodenum }-v \mid}+u}+c * \frac{1}{\sqrt{\text { Lifetime }}+u}
$$

The distance is the grid number from the node to the target grid, that is, the node number in the cluster grids. Lifetime is the survival time of the cluster grid, $a, b$ and $c$ as the weight number, and $a+b+c=1$. $U$ is in order to avoid the differentials of the calculated value overlarge, reference value set as $5 . \mathrm{V}$ is the node number of the cluster which has greater attract to the dissociative node, $10-15$ in general.

After confirming the target grid, the node will randomly select any location in the grids as the moving destination, and will randomly select any speed among (Minspeed,Maxspeed), to move to the destination.

\section{Pause}

After the node reaching to the destination, it will pause for a while. In order to make the model simple and easy to realize, the pausing time will be also randomly chosen among (Minpause,Maxspeed). After the pausing time, the node will re-select a new destination to repeat the process in (2) until the simulation terminates.

\section{THE EVALUATION OF CLUSTER EVOLUTION}

\section{MOBILITY MODEL}

This chapter will evaluate the cluster evolution mobility model, verify whether the model has the same cluster evolution character with the taxi GPS dataset, in order to guarantee the authenticity of the mobility model proposed in this paper. The following is basic parameter setting in the simulation experiment: 
Simulation tine: 10000, total node number:2000,

Minimum number of the cluster :5, simulation area: $x$ Range $* y$ Range $=10000 \mathrm{~m} * 10000 \mathrm{~m}$, Communication radius: $200 \mathrm{~m}, \alpha=0.2, \beta=0.3$,

(Minspeed,Maxspeed $)=(5 \mathrm{~m} / \mathrm{s}, 20 \mathrm{~m} / \mathrm{s})$,

$($ Minpause, Maxspeed $)=(10 \mathrm{~s}, 50 \mathrm{~s})$ 。

\section{A. Cluster size distribution}

In the cluster evolution mobility model, the cluster size distribution graph of different timing is shown as the following Fig1(a), it is observed from the graph that the complementary cumulative distribution function of the cluster size in different timing submits to the power-law distribution, that is, most of the cluster's node number is much smaller. From the Fig1(b), the complementary cumulative distribution function of the cluster size of the smooth model doesn't submit to the power-law distribution.
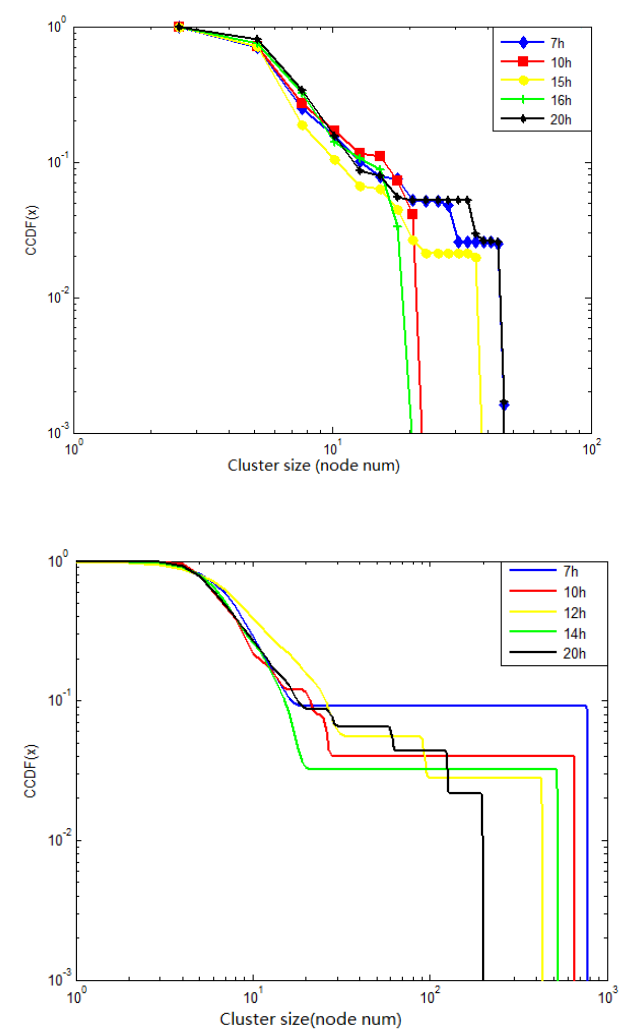

Figure 1. Complementary cumulative distribution function of the cluster size of the CEMM(a) and smooth(b)

\section{B. Division probability of clusters}

According to the analysis result of Shanghai taxi dataset, the tendency probability of the cluster division first increases then decreases along with the cluster node number, the division probability is the highest when the cluster size is about 10-15. Fig2 has shown the division tendency probability of the cluster evolution mobility model, the cluster evolution mobility model(in red curve) is in accordance with the increase and decrease trend of the Shanghai trace(in blue curve), which shows that the cluster evolution tendency of the mobility model proposed in this paper conforms with the practical situation. The division probability of the smooth model(in green curve) doesn't decrease as the cluster node number increases.

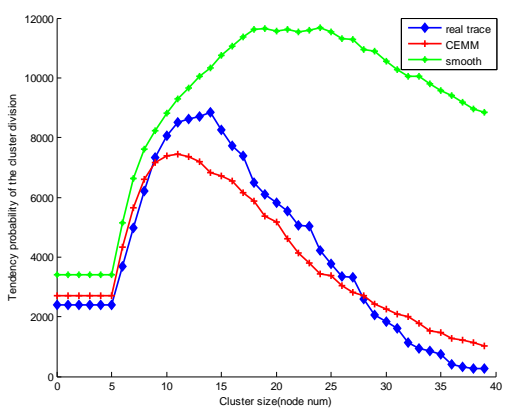

Figure 2. Tendency probability of the cluster division

\section{The change in the number of total cluster nodes}

The total cluster node number keeps dynamic stability in the cluster evolution process, that is, the node number joining in the cluster approximates the node number leaving the cluster. The variation trend of the cluster node in the cluster evolution mobility model is shown as the Fig3, the cluster node number keeps at the range of 300 or so, and is in a dynamic equilibrium. But in Fig4, the cluster node number decreases gradually in the smooth which is not in line with the practical situation.

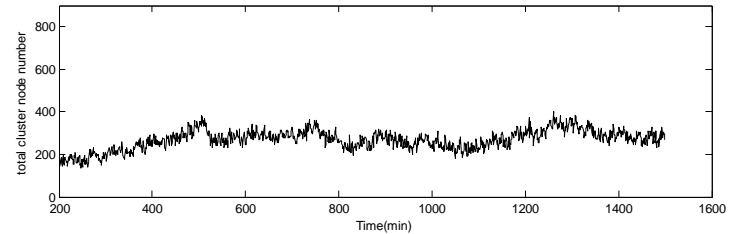

Figure 3. The change in the number of total cluster nodes in CEMM

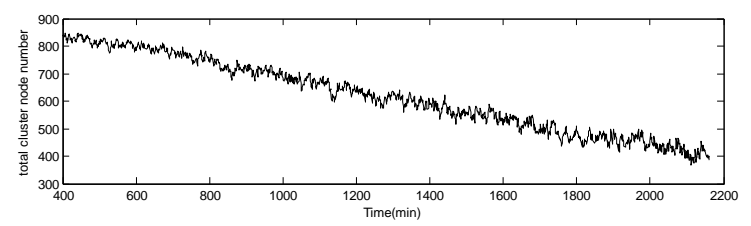

Figure 4. The change in the number of total cluster nodes in smooth 
D. The complementary cumulative distribution of the cluster's lifetime

Through the analysis result of the Shanghai taxi dataset, most of the cluster's lifetime is short, only little part of the cluster can maintain a longer lifetime. Seen from the fig5, the complementary cumulative distribution (in red curve) of the cluster's lifecycle in the mobility model proposed in this paper submits to the power-law distribution, which is in line with the analysis result of the practical dataset.

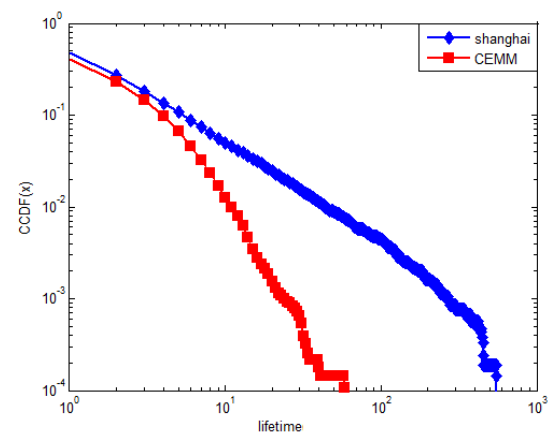

Figure 5. Complementary cumulative distribution of the cluster's lifetime

The above simulation result shows that the cluster evolution mobility model proposed in this paper is generally in accordance with the cluster evolution character of the practical dataset, which can factually reflect the cluster evolution discipline of the human mobility.

\section{CONCLUSIONS}

As to the analysis result of the cluster evolution character of the taxi's GPS dataset in this paper, a new mobility model has been proposed, which is based on the cluster evolution character (CEMM, Cluster Evolution based Mobility Model). In order to verify the authenticity of the mobility model proposed, this paper compares the cluster evolution mobility model and the taxi's practical GPS data with the classical human mobility character model, the smooth model. The result shows that in the aspect of the cluster size distribution, the cluster division trend and the cluster's node number, the smooth model is different with analysis result of the practical data, however, the mobility model proposed in this paper can well accord with the basic character of cluster evolution.
A further work will be put on the analysis of wireless router treaty's property under the cluster evolution mobility model, which will be compared with the classical model and the practical trajectory dataset. At the same time, verify the recently proposed vehicle-mounted and self-organized network mobility model and much more vehicles' trajectory dataset, apply the cluster evolution discipline to the vehicle-mounted and self-organized network mobility model.

\section{ACKNOWLEDGMENT}

This work was partly supported by National Natural Science Foundations of China (No. 61272061 and No.61301148), the fundamental research funds for the central universities of China (No.531107040263, 531107040276), the Research Funds for the Doctoral Program of Higher Education of China (No. 20120161120019 and No. 20130161110002), Hunan Natural Science Foundations of China (No. 10JJ5069 and No. 14JJ7023) and the Open Fund Project of Key Laboratory in Hunan Universities (No. 11K017).

\section{REFERENCES}

[1] M Grossglauser, D Tse.Mobility increases the capacity of ad hoc wireless networks, IEEE/ACM Transactions on Networking, 2002, 10(4), 477-486.

[2] F Bai, N Sadagopan. A Helmy.IMPORTANT: A framework to systematically analyze the Impact of Mobility on Performance of RouTingprotocols for Adhoc NeTworks. INFOCOM 2003 : 825-825.

[3] A B McDonald, T F Znati. A Mobility-based Framework for Adaptive Clustering in Wireless Ad Hoc Networks.In IEEE Journal on Selected Areas in Communications, vol. 17, issue 8, 1999 : 1466-1487.

[4] Dong Wang, Yong Zhang, Xiao-Hong Li. Cluster Dynamics Analysis of Human Mobile Network in Urban Environment. 10th International Conference on Wireless and Optical Communications Networks (WOCN2013), 2013.

[5] Nils Aschenbruck, Aarti Munjal, Tracy Camp.Trace-based mobility modeling for multi-hop wireless networks. Computer Communications,, 2011 : 704-714.

[6] B Liang, Z J Haas. Predictive distance-based mobility management for multidimensional pcs networks. IEEE/ACM Transactions on Networking, 2003, 11(5):718-732.

[7] Zhenguo Du, Peilin Hong, Kaiping Xue. Hotspot Gravitation Mobility Model, Communications Letters, 2012 : 193-195.

[8] K Lee, S Hong, S J Kim, I Rhee, S Chong. SLAW: A new mobility model for human walks. In Proceedings of IEEE INFOCOM, 2009 : 855-863

[9] Aarti M, Tracy C.SMOOTH: A Simple Way To Model Human Walks. Mobicom , 2010.

[10] M Musolesi, C Mascolo.Designing mobility models based on social network theory, ACM SIGMOBILE Mobile Computing and Communications Review, 2007, 11(3):59-70.

[11] Injong, R., S. Minsu, H. Seongik, L. Kyunghan, J.K. Secong and C. Song, 2011. On the levy walk nature of human mobility. Trans. Networking, 19: 630-643. 